\title{
Taking OWL to Athens
}

\section{Semantic Web Technology Takes Ancient Greek History to Students}

\author{
Jochen Reutelshoefer $^{1}$, Florian Lemmerich ${ }^{1}$, Joachim Baumeister ${ }^{1}$, \\ Jorit Wintjes $^{2}$, and Lorenz Haas $^{2}$ \\ 1 Institute of Computer Science, University of Würzburg, Germany \\ \{lastname\}@informatik. uni-wuerzburg.de \\ 2 Institute of Ancient History, University of Würzburg, Germany \\ \{firstname.lastname\}@uni-wuerzburg.de
}

\begin{abstract}
The HermesWiki project is a semantic wiki application on Ancient Greek History. As an e-learning platform, it aims at providing students effective access to concise and reliable domain knowledge, that is especially important for exam preparation. In this paper, we show how semantic technologies introduce new methods of learning by supporting teachers in the creation of contents and students in the personalized identification of required knowledge. Therefore, we give an overview of the project and characterize the semi-formalized content. Additionally, we present several use cases and describe the semantic web techniques that are used to support the application. Furthermore, we report on the user experiences regarding the usefulness and applicability of semantic technologies in this context.
\end{abstract}

\section{Introduction}

Students today are used to collect a large amount of required knowledge from the internet. While the number of available webpages is huge for almost any given topic, the quality of these pages is quite heterogeneous. Additionally, it is very difficult for students, especially undergraduate students, to extract the essential knowledge from extensive webpages. Therefore, students can significantly benefit from a reliable and concise knowledge base, that combines the advantages of traditional text books with the advantages of internet-based knowledge platforms, like accessibility, integration of multimedia resources, and the ability to update the contents easily. However, the creation of such a web application still is a tedious task, as texts and resources must be created, collected, and adapted to the context. Additionally, with the growing amount of content it again gets more difficult for students to find exactly the resources they require. In this paper, we describe how semantic technologies help to alleviate both problems in an e-learning project in the domain of Ancient Greek History, the HermesWiki.

The HermesWiki is a semantic wiki, developed to give students of history an introductory domain overview. As a full featured wiki system, HermesWiki allows for an effective collaborative composition of content. Furthermore, semantic

L. Aroyo et al. (Eds.): ESWC 2010, Part I, LNCS 6088, pp. 33\$-347, 2010.
(c) Springer-Verlag Berlin Heidelberg 2010 
technologies provide advanced functionality that generates additional value to the learning environment [1]: Formalized paragraphs and semantic annotations of the main text enable the contributors to easily generate additional content tailored to different tasks, such as tables of events, maps, or a quiz. Further, students have access to different forms of knowledge like narrative elements, ancient source texts, geo data, chronologic information and images. They can use semantic navigation to easily find the concepts they are looking for, filter the presented content by its relevancy on demand, and query the systems knowledge using an intuitive user interface.

The rest of the paper is organized as follows: In Section 2 we give an overview of the Hermes project and its context of use. Section 3 presents the underlying semantic model of the content that is created by the semantic wiki extension and discusses the Hermes ontology. Further, the use cases and the employed semantic technology are explained. Then, our experiences within the Hermes project are discussed. We present the technical basis of the implementation in Section 4 and conclude by presenting our ideas for future directions.

\section{HermesWiki - An Overview}

The HermesWiki is developed by the historians of the Department of Ancient History from the University of Würzburg, Germany, supported by the Department of Intelligent Systems of the Institute of Computer Science. The main purpose of the HermesWiki is to provide a concise and reliable overview of Ancient Greek History for teaching purposes of students. The project started in summer 2008 and its content is continuously growing since then. From the next semester on, the wiki is recommended as an alternative to traditional text books to all undergraduate students of the University of Würzburg, that take courses in Ancient History.

\subsection{Project History}

The development of the HermesWiki project was rather unusual compared to typical semantic application projects. The focused 'knowledge engineers' were historians with no experience in semantic technologies or knowledge management solutions. Initially, a web-based, easy to use content-management system, that supports collaborative development, was intended in order to structure e-learning content. Thus, at first a standard, non-semantic wiki was chosen as the favorable tool, since it was already known as a simple and flexible application. It soon became clear that this approach was a success as the wiki was quickly filled by a large knowledge base consisting mainly of textual content enriched with figures and an informal taxonomy for organizing the articles. Several intended use cases and the various possibilities of how to access and present the available content motivated the step-wise introduction of semantic technologies - possible due to the use of a semantic wiki [2]. 


\subsection{The Content: Knowledge about Ancient Greek History}

Currently, the HermesWiki contains about 700 pages which are written in German language. In general, we distinguish the following four categories:

1. A set of about 20 essays forms the core of the project. These essays of about 1,000 to 2,000 words each have been written especially for this project and provide a comprehensive overview of Ancient Greek History. Each essay covers one topic of the domain and is structured by a standardized template. Figure 1] shows the essay about Tyrannis. It includes a short list of the most important events generated from the ontology, an introductory section, that explains the context of this essay, a text description of the respective topic, an overview of ancient historical sources, and a formalized set of relevant events.

2. An extensive glossary of more than 500 articles provides students a short, but precise explanation of the used terminology. Described concepts include persons, geographic locations, and domain specific terms ("Autarky", "Polis", etc...) .

3. Around 60 translated excerpts of the referenced ancient sources are included in the wiki as separate wiki pages. Due to copyright reasons mostly own translations are used.

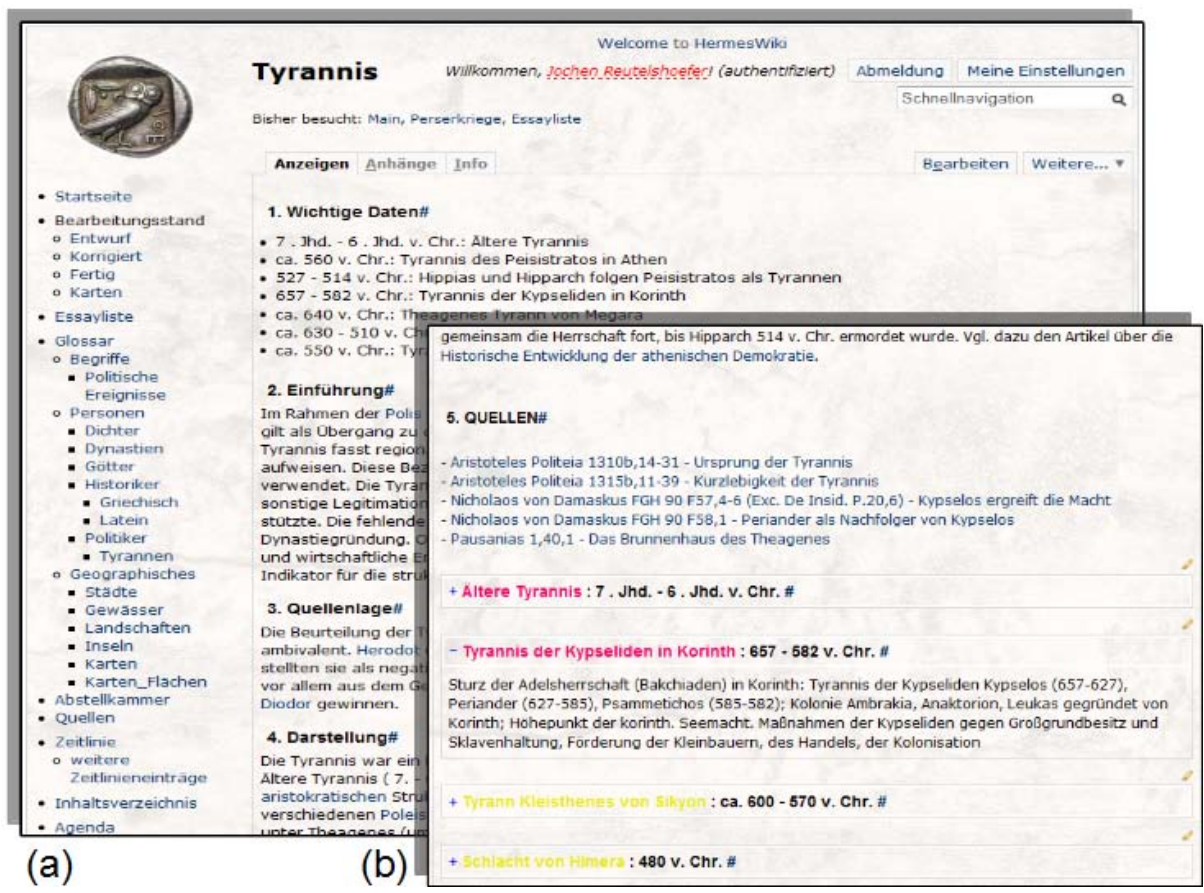

Fig. 1. The top (a) and bottom (b) of the wiki page containing the essay about Tyrannis taken from the HermesWiki. (Screenshots of the system, in German language). 
4. The organization and administration is supported by several wiki pages. These include "todo-lists", authoring guidelines, technical tutorials, bug reports, and separate pages for specialized tools, e.g., for wiki-wide refactoring of concept names or (semantic) search.

\subsection{The HermesWiki Community}

The contents of HermesWiki are contributed by the group of Ancient History of the University of Würzburg. The team consists of about a dozen persons: One professor, an assistant professor, their research staff, and a few selected graduate students, which work in close cooperation. These users have basic knowledge about computers and wiki systems like wikipedia, but no special experience with semantic wikis or semantic technologies in general. While the relatively small size of the community obviously limits the amount of contributions in the wiki, the high educational level of the authors guarantees a consistently high quality of the content.

In practice, the professor and the assistant professor of the team approve the content of each wiki page before release. Thus, typically a new article is created as follows: The professor/assistant professor identifies the need of an additional article and assigns this task to a team member. He/she collects necessary information, writes the article, and tags the respective wiki page as "draft". Afterwards, the article is proof-read by the professor/assistant professor, who either returns the article to the original author with suggestions for improvements, improves it by himself, or approves the page by tagging it as "revised".

\section{Social Semantic Technology for Ancient History}

In this chapter, we first present some main aspects of the ontology developed in the HermesWiki. Next, the knowledge formalization process that helps us to obtain formalized knowledge from the content entered by the contributors is described. Afterwards, we give some examples, how the ontology is used in the HermesWiki for the augmented presentation of the content, semantic navigation and search, and interactive sessions.

\subsection{The Hermes Ontology}

As already mentioned the project was not intended to be a "semantically enriched" wiki application from the beginning. However, the authors by themselves began to create categories and hierarchies for the domain concepts like persons, locations, and events to organize the contents. Thus, for the creation of a domain ontology of the HermesWiki we had to consider several requirements. We needed to integrate the informal conceptualization already given implicitly by the content. Further, we had to enrich it to support the planned use cases of the e-learning application. To avoid risky barriers for the domain experts, it seemed 
reasonable to keep the ontology as simple and sparse as possible with respect to the intended use cases, as the historians are no trained ontology engineers.

The starting point of the HermesWiki domain ontology was inspired by the VICODI project [3], which also developed an ontology for the historical domain. Considering the requirements stated above we had to conduct several modifications. We followed the KISS principle (keep it simple, stupid) — which is in line with the wiki spirit - to obtain an ontology, that is easy to understand for the historians and at the same time suits our formalization requirements for the intended use cases. For example, the VICODI ontology heavily relies on the role pattern. Thus, a person fulfills a role, e.g., is a king for a fixed period of time. For our applications this precise approach appeared to be unnecessarily detailed, so it was omitted. Figure 2(a) shows an excerpt of the resulting Hermes ontology. The upper part shows some exemplary concepts from the system ontology (wiki object model) sketching how the Hermes domain ontology is embedded into the wiki system. That is, each HermesConcept 'isDefinedBy' a TextOrigin allowing to trace the location where the concept has been defined in the wiki text. This can be done, e.g., by a wiki page, a single paragraph or an annotation markup.

Below, the concept hierarchy for the historical data is shown. Unlabeled links define subclass relations between two concepts represented by the connected nodes. Main concepts of the VICODI ontology, such as Individual, Location, Event, Social Stratum, and Ethnical Group have been reused and integrated with concepts proposed by the domain experts. In the center of the figure, the concept Event is depicted, which plays a key role in the knowledge formalization of HermesWiki. To each event a TimeStamp is attached containing the (possibly vague or uncertain) date information of the event. Further, a title and an importance rating (regarding exam relevance) is specified. Events can be connected to other concepts by object properties like involves or takesPlaceAt.

These properties as well as the taxonomies can also be edited or created by users inside the wiki using dash-tree markup [4. Any term being a dashtree child, i.e., follows with an incremented number of dashes, is defined as a subclass of its parent. For instance, in Figure 2(b) Dynasty and Social Stratum are defined as subclasses of Group of Persons. Since ontology changes can have a deep impact on some wiki features, they are only performed by experienced contributors. Major changes in the ontology are discussed in the community in advance.

Generally, the ontology is not intended to cover as much of the domain knowledge or wiki content as possible but only contains knowledge that is necessary for advanced features. In the next sections, we discuss how this ontology is populated by the domain experts of HermesWiki by editing and annotating wiki pages.

\subsection{Knowledge Formalization}

The methods of knowledge formalization in HermesWiki were designed considering three aspects: First, the way the historians intuitively organized the domain knowledge in the wiki in a natural way. Second, what formalized relations are 


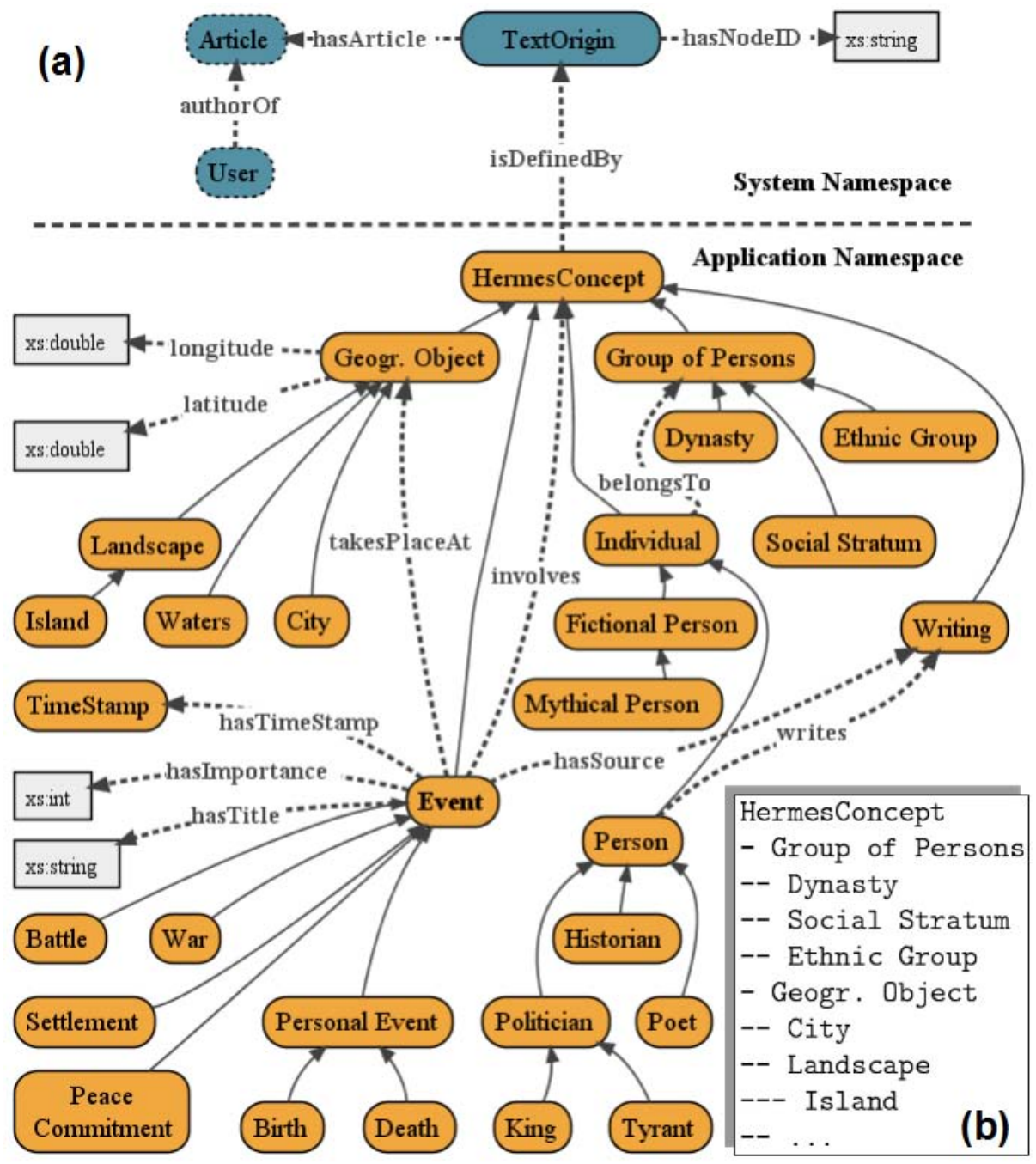

Fig. 2. (a) The Hermes Ontology (lower part) is connected to the system ontology (upper part). This figure is limited to the most important concepts of the project. (b) An example for dashtree markup for concept hierarchies.

required by use cases providing additional value to the e-learning application. Third, what are the additional costs, i.e., the workload, of this formalization. The content was continuously 'semantified', when an added value existed by enabling additional use cases.

The most common way to populate the ontology in semantic wikis is by annotating a page to represent an instance of some class. A large part of the knowledge in HermesWiki complies with this reasonable approach. However, often multiple relevant concepts are defined on one wiki page, when the 'one 
page - one instance' granularity is inappropriate, e.g., for events or geographical objects. As examples of knowledge formalization used in HermesWiki, we present a markup for the definition of geographical objects and time events. Further, we introduce the semi-automated workflow to connect the time events with other entities of the concept hierarchies like persons and geographic objects.

Definition of geographical objects. An important issue in the study of history is to set locations of historical events into context. As obtaining specialized maps can be a tedious (and costly) task, the HermesWiki offers easy to use possibilities to mark important places in maps. The longitude and latitude of geographic objects can be specified by a markup anywhere on any wiki page. For example the following (translated) markup specifies the geo-coordinates of the ontology instance "Marathon":

<<COORDINATES: Marathon, $38^{\circ} 9^{\prime} \mathrm{N}, 23^{\circ} 57^{\prime} \mathrm{E}>>$

This markup can also be obtained by using a Google Maps based editor in the wiki, that allows for the creation of the markup by specifying the concept name and clicking the respective location in the displayed map. The editor also features automatic search using the provided API.

Specifying time events. Time events are the most important concepts in the HermesWiki knowledge base. Like sketched in Figure 2, time events can connect the individuals of the concept hierarchy. In the wiki, time events often are only described by a couple of sentences and are closely interrelated. Thus, it seemed not pragmatic to create a separate page for each of these events, but to allow the definition of multiple events in one page. To formalize these time events in a flexible way (at any place of some wiki page), a markup was designed in cooperation with the historians. The entire definition of one time event is enclosed by the brackets ' $<<$ ' and ' $>>$ '. The following (translated) markup example describes the event Lamian War. As the first information the title of the event is given. It is followed by the importance rating defining its relevance for student exams. For instance, events with an importance rating of '1' are considered essential, while events with a rating of ' 3 ' are categorized as "additional knowledge". In the next line, the timestamp of the event is notated. The historians introduced a simple language for the definition of time intervals, which also includes annotations for different degrees of uncertainty (possibly resulting of inconsistent descriptions in different historical sources). The string " $323 \mathrm{~b}-322 \mathrm{~b}$ " points out, that the event occurred from $323 \mathrm{BC}$ until $322 \mathrm{BC}$. Next, the body of the markup follows consisting of a (free text) description of the actual event. The markup concludes with an (optional) list of historical sources where the event is mentioned, explicitly marked by the (translated) keyword "SOURCE:" as the first word of a new line (from Diodor and Pausanias in this example). 
$<<$ Lamian War (2)

$323 b-322 b$

After Alexanders death the Greeks revolted against

Macedonian rule under the lead of the Athenians.

$[\ldots .$.

SOURCE: Paus : :1, 25,3-6

SOURCE: Diod: : $18,8-18$

$>$. .

When the wiki page is saved, the time events are parsed and translated into the corresponding OWL concepts introduced in Figure 2, For each event an instance of type Event is created and the additional information is stored using the provided properties.

A semi-automated workflow for annotating time events. Events, locations, persons and other ontology instances, can be connected by adding appropriate relations into the ontology. This can be done by using a markup inside the wiki source text. However, to minimize the workload of the contributors, we additionally introduced a semi-automatic approach: Generally, the idea of semi-automated annotation in semantic wikis [25] is to use the background knowledge already given in the ontology, while processing the wiki text content and to propose probable relations to the user for confirmation. For the context of time event descriptions we assume, that any person or location important for this event is mentioned in its description. As those concepts are already present in the ontology they can be searched for (by name) in the description texts while parsing the time event markups. Although concept names in the event description strongly indicate a relation between the event and the respective concept, we use a semi-automated workflow to specify the kind of relation and to strictly avoid false positives, which is essential for the quality requirements of the project. Thus, whenever a concept is found, it is highlighted in the rendering of the event. Clicking on it will open up a popup window, which proposes some relations between the event and the mentioned concept, see Figure 3 . The possible relations are suggested, in this case takesPlaceAt and involves, together with the default options concept mismatch and do not ask again. These proposals can either be confirmed or discarded by the domain experts. The relations are proposed by inspecting the domain and range definitions of the properties in the ontology. If, for example, the name of an instance of class Person is found in the text description of the event, all properties with Event as domain and Person as range are proposed. Thus, dynamic definition of new properties is supported by this approach. When a relation is confirmed by clicking an option in the popup, an annotation markup of the phrase is added to the wiki source text. The corresponding OWL relation is generated while processing the annotation markup within the wiki source update. 


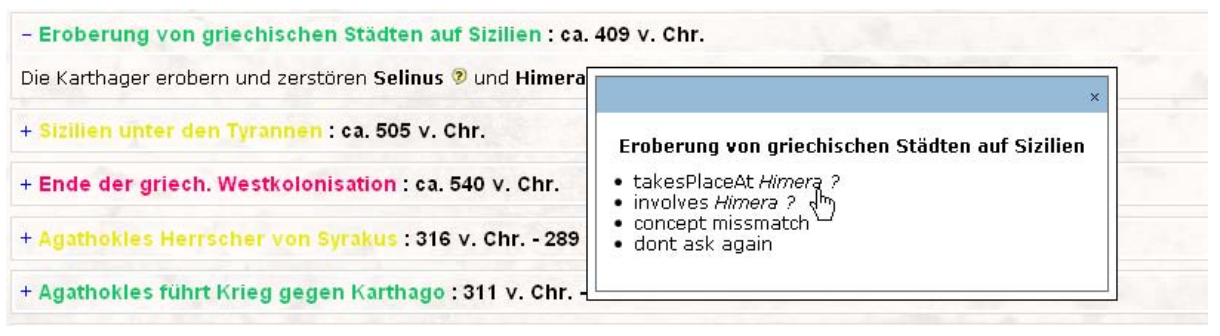

Fig. 3. Popups propose relations between the entities described in the text

The presented knowledge formalizations are extensively used in HermesWiki. For example, the ontology contains currently about 700 formally defined time events, 140 persons and 100 cities (with geo-coordinates), covering the knowledge in the intended scope of the project.

\subsection{Use Cases}

In this section we introduce use cases of semantic technologies. These are enabled by the advanced formalization features of HermesWiki and provide additional value for teachers and learners.

Inline queries for events. In HermesWiki the concept of inline queries, which is a common and beneficial feature of semantic wikis (e.g., [6]), has been adapted for filtering and displaying time events. Events are usually rendered at that place where they are defined but it might be didactically valuable to display specific selections of events in other contexts. For example, at the top of each essay a bullet list of the most important time events defined at the respective wiki page is displayed as depicted Figure 1(a). This can be done by using a flexible parameterized inline query mechanism. Inserting the respective tag into the wiki page sources allows to display events, that are related to some specific entity (e.g., Person, Geogr. Object), occurred in a defined period in time and/or have a minimum level of importance. For a seamless integration with full text descriptions, one can also specify the visualization of the events (e.g., as bullet lists or collapsible panels, with or without description).

Browsing through time - The Hermes Event Browser. While the inline queries allow to dynamically present selected time events in specific contexts to the students, the Hermes Event Browser enables the students themselves to search for time events by specifying a time interval. Additionally the search can be restricted to time events, that involve specific ontology instances, e.g, a Person or a Geogr. Object. The knowledge base is queried for time events fulfilling these constraints and the events are displayed, also providing links to related content.

Biographical timelines and maps. The wiki pages representing a person in most cases only contain a free text description with no further formalization. 
However, the extended knowledge formalization of time events, which connects the events with the involved persons, allows to provide additional (generated) information on these pages. Appended to the content of the page we generate a visualization of a curriculum. Querying the ontology allows to find events involving the respective person. These events are displayed in chronological order.

Furthermore, we retrieve the locations of the events assuming that the events had been annotated accordingly. With this data, HermesWiki is able to render a map, where all locations of events involving this person are inserted as markers at the location of its occurrence. Hence, the generated map illustrates a complete overview of the lifeline and the travels of this character. The marker of an event gives more detailed information about the event and provides a link to the text passage in the wiki, where the event is defined and described. Figure 4 shows the wiki page of Alexander the Great containing a generated map displaying the locations of the most important events in the life of Alexander the Great.

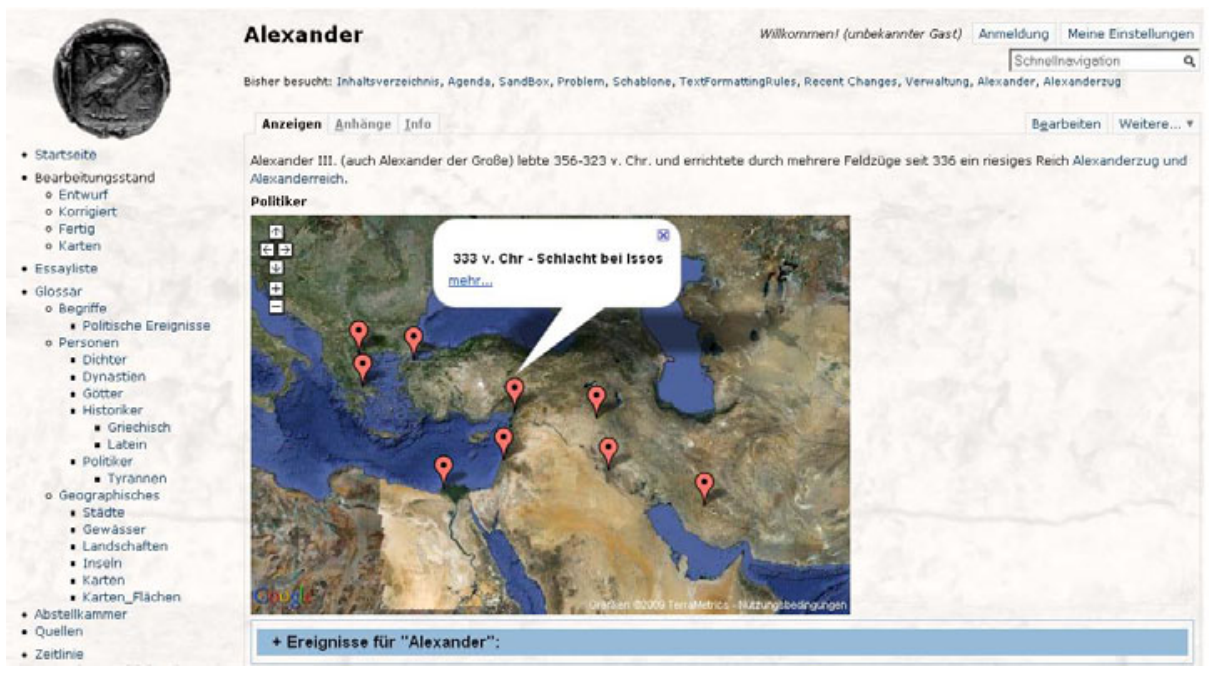

Fig. 4. The wiki page of Alexander the Great. It contains a map generated by the information in the ontology. It shows the most important events in the life of Alexander.

The HermesQuiz. The Hermes-Quiz is an interactive component, where students can answer (multiple-choice) questions to test their knowledge. The questions are generated by the system, based on the formal relations contained in the ontology. For example, the ontology contains the information, that the beginning of the Persian empire was about $552 \mathrm{BC}$. The system can generate two other dates (taken from some other randomly chosen time events) and pose the correct and the generated dates as a three-choice question. As depicted in Figure 5 the quiz panel shows a new question (lower box) and waits for the user to choose the correct date. In the upper box the solution of the last answered question is presented, also containing a link to the description of the corresponding time 
event. At the bottom, the score for the current quiz session is displayed, that is, the ratio of correctly answered questions.

The quiz could be extended in the future to ask for other types of relations contained in the ontology, however, generating good questions can be a nontrivial task in general (see for instance [7]).

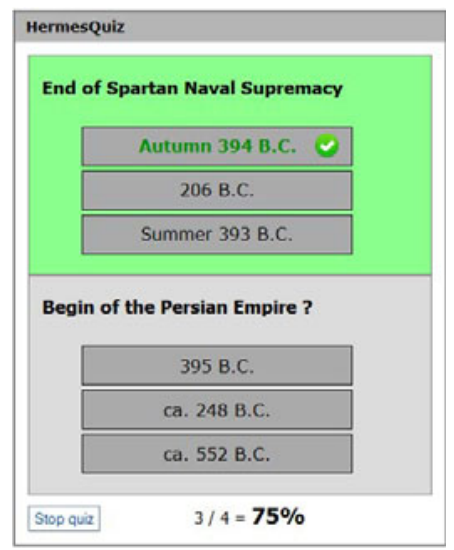

Fig. 5. The Hermes Quiz allows the user to test his knowledge with questions generated from the ontology

\subsection{Experiences}

Next, we describe some experiences on the usage of semantic technologies and the workflow in the HermesWiki community.

Experiences using semantic technology. In the context of this application we applied the approach of a Decorated Semantic Wiki 8 . This concept tackles the barrier for untrained users to contribute to semantic applications by hiding the semantic technology, considering formalization and use, behind 'interfaces' designed especially for the domain and user community. These interfaces are provided by formalization methods like special markups or popup questions and are tailored to the users comprehension of the domain and the data. They allow users with only little experience in ontology 1 engineering or its technical implementation, to contribute. Wikis aim to provide the lowest entry barrier possible and this extension of the wiki philosophy towards semantic data appears to be successful in our project, considering the high formalization level of the HermesWiki contents.

In this project, the use of semantic technologies such as OWL and SPARQL proved to be a very suitable basis for fulfilling the (extended) application requirements. The flexible knowledge repository in combination with the wiki allows for

${ }^{1}$ The term ontology exists in humanities considerably longer than in computer science, which can lead to misunderstandings in this domain. 
incremental formalization of the content as well as for modifications/extensions in the ontological schema. Considering the pathway of the project, where the demand of advanced features developed step by step over time without predefined requirement specification, the use of semantic technology proved to be much more flexible at the implementation level when compared to conventional techniques. It appears that any (new) feature can be implemented fairly independently from the other using the ontology, superseding time-consuming changes in database or object schemas.

By utilizing an extensible semantic wiki as core component, the development could concentrate on domain and application specific issues, thus strongly limiting the implementation efforts.

Workflow experiences. When starting a knowledge engineering application with domain experts, it is always reasonable to integrate methods and tools that the domain experts are already familiar with. For example, in the VICODI project the ontology was populated by uploading Excel spreadsheets [3]. The contributors of our project are heavily using standard office software for other work. When the task of adding a new topic to the wiki is assigned to an author, the first version is often written offline in an editor of his choice. Then it is pasted into the wiki and tagged as 'draft'. After that, the community is invited to review, refine, extend and 'semantify' this article. This employed process model complies with the idea of Seeding and Evolutionary Growth in collaborative knowledge acquisition proposed by Fischer et al. 9]. Considering the total amount of content in the HermesWiki, this procedure yields a relatively small number of user-edits (about 6200 at the time of writing).

Most of the time, team members spend only a few hours a week working on HermesWiki. However, about twice per year the complete team meets for a half week workshop. In these workshops, major decisions of the project and new software features are discussed. The rest of the time is dedicated to the creation and improvement of wiki contents. This also showed to be beneficial for system developers, as it allowed to get insights of user behavior and thus identify helpful system improvements.

\section{Implementation}

The HermesWiki is a plugin for the semantic wiki KnowWE [10, which itself is a semantic extension of JSPWiki, an open source wiki engine implemented in Java2. For the management of RDF-data, KnowWE employs the Sesame framework 3 and (swift-) OWLIM, which also supports rule-based reasoning (e.g., of OWL-Horst rules [1]). The KnowWE system, bringing semantic wiki features for formalization and querying of knowledge out of the box, is primarily designed for extensibility [8]. The design concept aims to support customization

\footnotetext{
2 http://www.jspwiki.org

3 http://www.openrdf.org/

4 http://www.ontotext.com/owlim/
} 
towards specialized semantic wiki applications, that then can provide domain and user specific features. The Hermes extension uses the KnowWE API and the plugin mechanisms to introduce the custom features discussed in Section 3. The components contained in the Hermes extension can be classified into the two categories formalization and presentation:

- Formalization: Contains the parser components to create the parse-tree of the page and translation functions generating OWL data (e.g., markup for time events) for markup used beyond the standard KnowWE markup.

- Presentation: Contains the components for querying and visualization implementing the features presented in Section 3.3 (e.g., inline queries, biography visualization and the quiz). These components typically contain some SPARQL query expression containing some placeholder, which is replaced before execution according to the current context of use (e.g., page name and user input). With the results of the 'coined' SPARQL query retrieved from the RDF-store the result is rendered for the user by common HTML/CSS/DOM-scripting techniques, also utilizing the Google Maps API.

The Hermes plugin as well as KnowWE itself is available under LGPL license and can be downloaded at https://isci.informatik.uni-wuerzburg.de.

\section{Related Work}

Several general approaches using semantic technologies for e-learning have been proposed before, compare e.g., [1. However, in humanities, especially history, semantic technologies have only received little attention with respect to e-learning applications so far. The Perseus Project5 for example provides a freely available digital library, including source texts and multimedia content for Ancient Greek History. However, in contrary to HermesWiki it does not offer concise descriptions of historical events and is not using semantic technologies at all. On the contrary, within the VICODI project [3] a detailed ontology for the domain of history has been developed. However, this project was not focused on teaching or learning. While many concepts of the VICODI ontology could be reused for this project, major modifications were required for the deployment in our context of e-learning. In the related domain of cultural heritage documentation the CIDOC $C R M 6$ ontology with a slightly different scope has been developed.

Today for many domains, including history, general knowledge is collected by Wikipedia. In fact, it shows up that for most of the content of the HermesWiki there can be found some correspondence in the online encyclopedia. Much scientific work has been done reusing this vast accessible resource and processing the data for specific presentation, like for example done by Sipos et al. 12. There are several reasons why this approach is not applicable for the HermesWiki application: Although the methods to extract formal relations from informal text

\footnotetext{
5 WWW.perseus.tufts.edu

6 http://cidoc.ics.forth.gr/
} 
sources have made considerable progress during the last years (e.g., resulting in systems like DBpedia [13]), they still cannot guarantee the degree of correctness that is necessary in an e-learning scenario for exam relevant knowledge. Further, Wikipedia as a resource for scientific work is a heavily discussed topic. In the domain of history, often well established historic facts are mixed up with remarks lacking of historicity. Additionally, a main goal of the project is to provide a delimitation of the knowledge that needs to be learned for certain exams.

Schaffert et al. [14] propose the use of a semantic wiki in the e-learning context for combining the advantages of usability, knowledge formalization and full control of the contents. This approach complies with the spirit of our project.

\section{Conclusions}

In this paper we presented the benefits of semantic web technologies in the HermesWiki application, an e-learning project in the domain of Ancient Greek History. We provided an overview of the project itself and its community. Then, we explained how semantic technologies are employed to generate additional value for the application by describing the cycle of generation and use of formalized knowledge, and presented in-use experiences. Further, we briefly presented the implementation of the system and discussed related approaches. The HermesWiki project indicates that the additional employment of semantic technologies as an extension to well-established tools, and methods can generate considerable additional value at relatively low implementation costs. For future work, we plan to extend the quiz approach for asking general relations. To obtain even more formalized data we will evolve the semi-automated annotation approach.

As the domain overview on Ancient Greek History is approaching completion and HermesWiki shows to be successful, the community is also planning on extending the content of the wiki to Ancient Roman History. A table of contents and main time events have been already specified inside the wiki.

The HermesWiki is exclusively accessible for students of the University of Würzburg. Some exemplary pages will be released to the public under the URL http://hermeswiki.informatik.uni-wuerzburg.de.

\section{References}

1. Stojanovic, L., Staab, S., Studer, R.: eLearning based on the Semantic Web. In: WebNet 2001 - World Conference on the WWW and Internet, pp. 23-27 (2001)

2. Schaffert, S., Bry, F., Baumeister, J., Kiesel, M.: Semantic wikis. IEEE Software 25(4), 8-11 (2008)

3. Nagypál, G., Deswarte, R., Oosthoek, J.: Applying the Semantic Web - The VICODI Experience in Creating Visual Contextualization for History. Literary and Linguistic Computing 20(3), 327-349 (2005)

4. Baumeister, J., Reutelshoefer, J., Puppe, F.: Markups for Knowledge Wikis. In: SAAKM'07: Proceedings of the Semantic Authoring, Annotation and Knowledge Markup Workshop, Whistler, Canada, pp. 7-14 (2007) 
5. Ruiz-Casado, M., Alfonseca, E., Castells, P.: From Wikipedia to Semantic Relationships: a Semi-automated Annotation Approach. In: SemWiki06: Proceedings of 1st Semantic Wiki workshop - From Wiki to Semantics (2006)

6. Völkel, M., Krötzsch, M., Vrandecic, D., Haller, H., Studer, R.: Semantic Wikipedia. In: WWW '06: Proceedings of the 15th international conference on World Wide Web, pp. 585-594. ACM, New York (2006)

7. Zitko, B., Stankov, S., Rosic, M., Grubisic, A.: Dynamic test generation over ontology-based knowledge representation in authoring shell. Expert Syst. Appl. 36(4), 8185-8196 (2009)

8. Reutelshoefer, J., Lemmerich, F., Haupt, F., Baumeister, J.: An Extensible Semantic Wiki Architecture. In: SemWiki'09: Fourth Workshop on Semantic Wikis - The Semantic Wiki Web (CEUR proceedings 464) (2009)

9. Fischer, G.: Seeding, Evolutionary Growth and Reseeding: Constructing, Capturing and Evolving Knowledge in Domain-Oriented Design Environments. Automated Software Engineering 5(4), 447-464 (1998)

10. Baumeister, J., Reutelshoefer, J., Puppe, F.: KnowWE: A Semantic Wiki for Knowledge Engineering. Applied Intelligence (2010)

11. ter Horst, H.J.: Combining RDF and Part of OWL with Rules: Semantics, Decidability, Complexity. In: Gil, Y., Motta, E., Benjamins, V.R., Musen, M.A. (eds.) ISWC 2005. LNCS, vol. 3729, pp. 668-684. Springer, Heidelberg (2005)

12. Sipos, R., Bhole, A., Fortuna, B., Grobelnik, M., Mladenic, D.: Demo: HistoryViz - Visualizing Events and Relations Extracted from Wikipedia. In: Aroyo, L., Traverso, P., Ciravegna, F., Cimiano, P., Heath, T., Hyvönen, E., Mizoguchi, R., Oren, E., Sabou, M., Simperl, E. (eds.) ESWC 2009. LNCS, vol. 5554, pp. 903-907. Springer, Heidelberg (2009)

13. Bizer, C., Lehmann, J., Kobilarov, G., Auer, S., Becker, C., Cyganiak, R., Hellmann, S.: DBpedia - A crystallization point for the Web of Data. Journal of Web Semantics: Science, Services and Agents on the World Wide Web 7(3), 154-165 (2009)

14. Schaffert, S., Bischof, D., Bürger, T., Gruber, A., Hilzensauer, W., Schaffert, S.: Learning with Semantic Wikis. In: SemWiki'06: Proceedings of 1st Semantic Wiki workshop - From Wiki to Semantics (2006) 